\title{
What do I do now? Intolerance of uncertainty is associated with discrete patterns of anticipatory physiological responding to different contexts
}

Article

Accepted Version

Morriss, J. (2019) What do I do now? Intolerance of uncertainty is associated with discrete patterns of anticipatory physiological responding to different contexts.

Psychophysiology, 56 (9). e13396. ISSN 0048-5772 doi: https://doi.org/10.1111/psyp.13396 Available at https://centaur.reading.ac.uk/83879/

It is advisable to refer to the publisher's version if you intend to cite from the work. See Guidance on citing.

To link to this article DOI: http://dx.doi.org/10.1111/psyp.13396

Publisher: Wiley-Blackwell

All outputs in CentAUR are protected by Intellectual Property Rights law, including copyright law. Copyright and IPR is retained by the creators or other copyright holders. Terms and conditions for use of this material are defined in the End User Agreement. 


\section{CentAUR}

Central Archive at the University of Reading

Reading's research outputs online 
What do I do now? Intolerance of uncertainty is associated with discrete patterns of anticipatory physiological responding to different contexts Jayne Morriss*

Centre for Integrative Neuroscience and Neurodynamics

School of Psychology and Clinical Language Sciences

University of Reading

Reading

UK

${ }^{*}$ Correspondence:

Jayne Morriss

Centre for Integrative Neuroscience and Neurodynamics

School of Psychology and Clinical Language Sciences

University of Reading

Earley Gate, Whiteknights Campus

RG6 6AH Reading

United Kingdom

(0)118378 7937

j.e.morriss@reading.ac.uk 


\begin{abstract}
Heightened physiological responses to uncertainty are a common hallmark of anxiety disorders. Many separate studies have examined the relationship between individual differences in intolerance of uncertainty (IU) and physiological responses to uncertainty during different contexts. Despite this there is a scarcity of research examining the extent to which individual differences in IU are related to shared or discrete patterns of anticipatory physiological responding across different contexts. Anticipatory physiological responses to uncertainty were assessed in three different contexts (associative threat learning and extinction, threat uncertainty, decisionmaking) within the same sample $(n=45)$. During these tasks, behavioural responses (i.e. reaction times, choices), skin conductance and corrugator supercilli activity were recorded. In addition, self-reported IU and trait anxiety were measured. IU was related to both skin conductance and corrugator supercilii activity for the associative threat learning and extinction context, and decision-making context. However, trait anxiety was related to corrugator supercilii activity during the threat uncertainty context. Ultimately, this research helps us further tease apart the role of IU on different aspects of anticipation (i.e. valence and arousal) across contexts, which will be relevant for future IU-related models of psychopathology.
\end{abstract}

Keywords: Anticipation, Uncertainty, Intolerance of Uncertainty, Trait Anxiety, Skin Conductance, Electromyography 


\section{Introduction}

Individuals who are high in intolerance of uncertainty (IU) are described as having a 'dispositional incapacity to endure the aversive response triggered by the perceived absence of salient, key, or sufficient information, and sustained by the associated perception of uncertainty' (Carleton, 2016b, p 31). IU has been linked to many mental health disorders that have an anxiety component (Gentes \& Ruscio, 2011; McEvoy \& Mahoney, 2012). On this basis the study of IU has gained substantial momentum in recent years and now sits at the forefront of anxiety research (Grupe \& Nitschke, 2013; Tanovic, Gee, \& Joormann, 2018). Initial work shows that individuals high in IU, relative to individuals low in IU, display heightened physiological and neural activity to uncertainty across several different domains (for review see Tanovic, Gee \& Joorman, 2018). The most popular areas of research for examining uncertainty and IU have been in the following contexts: (1) associative threat learning and extinction, (2) threat uncertainty, and (3) decision-making (for review see, Morriss, Gell \& van Reekum, 2018).

There is robust evidence for the role of IU in associative learning, particularly when updating threat to safe associations, as in threat extinction (Chin, Nelson, Jackson, \& Hajcak, 2016; Dunsmoor, Campese, Ceceli, LeDoux, \& Phelps, 2015; Lucas, Luck, \& Lipp, 2018; Morriss, Christakou, \& Van Reekum, 2015, 2016; Morriss, Macdonald, \& van Reekum, 2016; Morriss \& van Reekum, 2019). The context of threat extinction is inherently uncertain, as at the start of extinction the contingencies are unknown. For example, after 100\% reinforcement, high IU, relative to low IU individuals have been found to show generalized skin conductance response and amygdala activity across threat and safety cues during early extinction, and to show continued skin conductance responding and amygdala 
activity to threat versus safety cues during late extinction (Morriss, Christakou, \& van Reekum, 2015, 2016).

The evidence for the role of $\mathrm{IU}$ in threat uncertainty tasks is mixed (Bennett, Dickmann, \& Larson, 2018; Gole, Schäfer, \& Schienle, 2012; Grupe \& Nitschke, 2011; Nelso \& Shankman, 2011; Schienle, Köchel, Ebner, Reishofer, \& Schäfer, 2010; Somerville et al., 2013). In these tasks the uncertainty and valence parameters are known, thus the goal of the participant is to tolerate the potential for an uncertain aversive event. For example, participants will be presented cues that predict either negative or neutral pictures (Grupe \& Nitschke, 2011; Somerville et al., 2013), or will follow a predictable or unpredictable countdown to a shock (Nelson \& Shankman, 2011). During these tasks, individuals high in IU, relative to low IU have been shown to exhibit: (1) heightened amygdala activity to cues conveying uncertainty (Schienle et al., 2010) and to aversive pictures following unpredictable countdowns (Somerville et al., 2013), and (2) reduced startle magnitude during the anticipation of temporally uncertain threat (Nelson \& Shankman, 2011). However, a number of threat uncertainty studies have not found significant relationships between IU and anticipatory physiological responses (Bennett et al., 2018; Grupe \& Nitschke, 2011).

There is a small emerging literature on the role of IU in decision-making. The decision-making tasks that have been used to examine IU vary substantially. In the majority of the decision-making tasks the uncertainty and valence parameters are known, and the goal of the participant is to make optimal decisions in order to gain reward and avoid loss. Individuals high in IU have been found to report more distress (Jacoby, Abramowitz, Reuman, \& Blakey, 2016; Jacoby, Reuman, Blakey, Hartsock, \& Abramowitz, 2017) and make more draws to decision on the beads task (Jacoby, Abramowitz, Buck, \& Fabricant, 2014; Ladouceur, Talbot, \& Dugas, 1997). In 
addition, a few studies have demonstrated that high IU individuals are more likely to choose immediate smaller rewards over waiting for larger rewards (Carleton et al., 2016; Luhmann, Ishida, \& Hajcak, 2011; Tanovic, Hajcak, \& Joormann, 2018). Taken together these findings suggest that individuals scoring high in IU will seek more information to reduce uncertainty and will not wait to make a decision when there is no additional information about the uncertain outcome.

Despite recent advances in understanding $\mathrm{IU}$, there is a scarcity of research examining the extent to which individual differences in IU are related to discrete or shared aspects of anticipatory physiological responding (i.e. valence and arousal) across different contexts. This makes it difficult to assess the generalizability or specificity of IU-related physiological profiles and their relevance to psychopathology models and aetiology (Shihata, McEvoy, Mullan, \& Carleton, 2016). Based on this, the following study attempted to fill in some of the gaps in the literature by measuring anticipatory physiological responses related to valence or arousal during different contexts: an associative threat learning task with acquisition and extinction phases; a threat uncertainty task with cues that predict negative or neutral pictures; a decisionmaking task where uncertain stimuli are categorised in the absence of reward or loss.

The associative threat learning and threat uncertainty tasks were similar to those that had already been designed to assess IU. Both these tasks included anticipated aversive outcomes, as previous literature using these tasks has focused on the interaction between IU, levels of uncertainty, and threat (Grupe \& Nitschke, 2013; Tanovic, Gee, et al., 2018). The associative threat learning and extinction task was uninstructed, leaving participants to learn and update the contingencies on their own. The threat uncertainty task was instructed, which meant that participants were 
given the contingencies before the task started. The decision-making task was designed to assess the anticipation of making a decision in the absence of valenced outcomes. The majority of previous studies on IU and decision-making have focused on reward and loss. However, it is unknown whether decision-making under uncertainty in the absence of valenced outcomes is aversive enough to modulate anticipatory physiological responses. Indeed, previous research has observed IU to modulate other psychological mechanisms such as attention in the absence of threat (Fergus, Bardeen, \& Wu, 2013; Fergus \& Carleton, 2016; Morriss \& McSorley, 2019).

Throughout the tasks anticipatory physiological responses were measured using skin conductance and corrugator supercilli activity. Skin conductance response is thought to capture arousal (Dawson, Schell, \& Filion, 2000) and corrugator supercilli activity is thought to capture valence (Tassinary, Cacioppo, \& Vanman, 2007). For example: (1) greater skin conductance response is observed for both negative and positive pictures, relative to neutral pictures, and (2) greater corrugator supercilli activity is observed for negative, relative to positive and neutral pictures (Bradley \& Lang, 2000). The advantage of recording both skin conductance response and corrugator supercilli activity is that it may reveal whether there are specific aspects of arousal and valence related to IU and anticipation during the different contexts.

Alongside the physiological measures, ratings of threat vs. safety cues were recorded for the associative learning task, and choices and reaction times were recorded for the decision-making task. In addition, self-reported IU and trait anxiety (STAI) (Spielberger, Gorsuch, Lushene, Vagg, \& Jacobs, 1983) were measured. STAl is a popular measure for self-reported anxiety and has been used in 
associative learning, threat uncertainty and decision-making literatures (Grupe \& Nitschke, 2013; Hartley \& Phelps, 2012; Lonsdorf \& Merz, 2017). To assess the specificity of IU during different contexts, it is useful to contrast it with other selfreported anxiety measures such as STAI (for discussion see Morriss, Christakou \& van Reekum, 2016).

For the associative learning task it was predicted that all participants would display greater expectancy ratings, skin conductance and corrugator supercilli activity to learned threat (CS+) vs. safety (CS-) cues during acquisition, and that the differential response to the CS+ vs. CS- for skin conductance and corrugator supercilli activity would reduce over time during extinction. Given previous research, it was predicted that individuals scoring higher in IU would show heightened skin conductance and corrugator supercilli activity during extinction (Morriss et al., 2015; Morriss, Christakou, et al., 2016; Morriss, Macdonald, et al., 2016). No predictions were made for IU and expectancy ratings during the associative learning task, due to the lack of findings in previous studies.

For the threat uncertainty task it was predicted that all participants would exhibit larger skin conductance and corrugator supercilli activity to: (1) cues predicting certain negative pictures vs. cues predicting uncertain pictures and cues predicting certain neutral pictures, and (2) negative pictures following certain cues vs. negative pictures following uncertain cues and neutral pictures following uncertain/certain cues. Moreover, it was predicted that higher IU would be associated with greater skin conductance and corrugator supercilli activity to the uncertain cue and to negative pictures following an uncertain cue, relative to the other conditions. 
Lastly, for the decision-making task, it was predicted that all participants would display relatively accurate choices based on probability. In addition, it was predicted that all participants would show larger reaction times, skin conductance and corrugator supercilli activity when making decisions on uncertain stimuli vs. certain stimuli. Furthermore, it was predicted that individuals scoring higher in IU relative to lower IU would display larger reaction times, skin conductance and corrugator supercilli activity when making decisions on uncertain stimuli vs. certain stimuli.

If IU is associated with valence and arousal related aspects of anticipatory physiological responding across contexts then IU likely serves to modulate both valence and arousal related anticipation generally. Alternatively, if IU is only associated with some valence and arousal related aspects of anticipatory physiological responding during some contexts then it suggests that IU may serve to modulate anticipatory mechanisms distinctly depending on the context.

\section{Method}

\subsection{Participants}

Forty-five volunteers $(M$ age $=23$ years, $S D$ age $=4.23$ years; 31 females and 14 males; 33 Europeans, 5 Asian, 4 African/Afro Caribbean, 3 Mixed) took part in the study. The sample size was based on previous experiments that have examined IUrelated differences in associative learning using psychophysiological measures (Chin, Nelson, Jackson, \& Hajcak, 2016; Lucas, Luck, \& Lipp, 2018; Morriss, Christakou, \& van Reekum, 2016; Morriss). All participants had normal or corrected to normal vision. Participants provided written informed consent and received $£ 10$ for their participation. Advertisements and word of mouth were used to recruit 
participants from the University of Reading and local area. No exclusion criteria were used (i.e. did not control for use of psychoactive medication, psychiatric or neurological illnesses). Two participants withdrew from the associative threat learning task and one withdrew from the threat uncertainty task. There was a recording error for one participant on the associative threat learning task. The procedure was approved by the University of Reading Research Ethics Committee.

\subsection{Procedure}

When participants arrived at the laboratory they were informed about the experimental procedures. Firstly, participants were seated in the testing booth and asked to complete and sign a consent form as an agreement to take part in the study. Secondly, participants completed questionnaires on a computer (see 2.4 below). Thirdly, electromyography sensors were attached to the left corrugator supercilli and physiological sensors were attached to the participants' left hand. The tasks (see 2.3 below) were presented in a counterbalanced order on a computer, whilst skin conductance, interbeat interval (not analysed) and behavioural ratings were recorded. Participants were instructed to maintain attention to the tasks and to stay as still as possible. The experiment took approximately 60 minutes in total.

\subsection{Tasks}

All tasks were designed using E-Prime 2.0 software (Psychology Software Tools Ltd, Pittsburgh, PA). Visual stimuli were presented at a $60 \mathrm{~Hz}$ refresh rate on an $800 \mathrm{x}$ 600 pixel computer screen. Participants sat approximately $60 \mathrm{~cm}$ from the screen. 
2.3.1 Associative threat learning task: The experimental design for the associative threat learning task was identical to previous work (Morriss \& van Reekum, 2019). Participants were required to passively view cues that predicted a threat or safe outcome. Participants did not receive instructions about the threat/safe contingencies and were simply told to pay attention to the squares and sounds.

Visual stimuli were blue and yellow squares with $183 \times 183$ pixel dimensions. The aversive sound stimulus was presented through headphones. The sound consisted of a threat inducing female scream used in previous experiments (Morriss et al., 2015; Morriss et al., 2016). The volume of the sound was standardized across participants by using fixed volume settings and was verified by an audiometer prior to each session $(90 \mathrm{~dB})$.

The task comprised of two learning phases: acquisition and extinction. Both acquisition and extinction consisted of two blocks each. In acquisition, one of the coloured squares (blue or yellow) was paired with the aversive $90 \mathrm{~dB}$ sound $50 \%$ of the time (CS+), whilst the other square (yellow or blue) was presented alone (CS-). During extinction, both the blue and yellow squares were presented in the absence of the US.

The acquisition phase consisted of 24 trials (6 CS+ paired, $6 \mathrm{CS}+$ unpaired, 12 CS-) and the extinction phase 32 trials (16 CS+ unpaired, 16 CS-). Early extinction trials consisted of the first $8 \mathrm{CS}+$ and CS- trials, and late extinction trials consisted of the last 8 CS+ and CS- trials. Experimental trials were pseudorandomised such that the first trial of acquisition was always paired and then after all trial types were randomly presented. Conditioning contingencies were counterbalanced, with half of participants receiving the blue square paired with the US and the other half of participants receiving the yellow square paired with the US. 
The coloured squares were presented for a total of $4000 \mathrm{~ms}$. The aversive sound lasted for 1000 ms, which coterminated with the reinforced CS+'s. Subsequently, a blank screen was presented for 6000 - 8000 ms (see Figure 1).

At the end of each block, participants were asked to rate how much they expected the blue square and yellow square to be followed by the sound stimulus, where the scale ranged from 1 ("Don't Expect") to 9 ("Do Expect"). Two other 9-point Likert scales were presented at the end of the task. Participants were asked to rate:

(1) the valence and (2) arousal of the sound stimulus. The scales ranged from 1 (Valence: very negative; Arousal: calm) to 9 (Valence: very positive; Arousal: excited).

\subsubsection{Threat uncertainty task:}

The experimental design for the threat uncertainty task was similar to previous work (Gole et al., 2012; Grupe \& Nitschke, 2011; Schienle et al., 2010). Participants were required to passively view cues that predicted certain negative, certain neutral or uncertain negative/neutral pictures. Participants were instructed as to which cue predicted a given outcome.

Cues consisted of white courier text with 48 font size (e.g. X, O, ?) presented centrally on a black background. The ' $X$ ' signified that the participant would receive a certain negative picture. The ' $O$ ' signified that the participant would receive a certain neutral picture. The '?' signified that the participant could receive a negative or neutral picture.

Thirty-six pictures from the international affective picture system (IAPS) were presented (Lang, Bradley, \& Cuthbert, 2005) (for picture list see Supplementary Material, Table 1). Half of the pictures comprised of negative content (i.e. war, 
accidents, mutilation, infants in distress) and the other half contained neutral content (i.e. work, children and adults in neutral settings, objects). Based on the original ratings from the IAPS battery, negative pictures were significantly more negative in valence and arousing than neutral pictures, $p$ 's $<0.001$. Negative and neutral pictures did not vary in complexity or luminosity, p's $>0.4$. Complexity was calculated from using the jpeg size of the images (Negative $=129222.7 ;$ Neutral $=121668.4$ ) and luminosity was calculated from using the RGB values of the images (Negative $=$ 0.369 ; Neutral $=0.375)$. Pictures were presented to fit the $800 \times 600$ pixel dimensions of the screen.

The task consisted of 36 trials (12 certain negative, 12 certain neutral, 6 uncertain negative, 6 uncertain neutral). Experimental trials were randomised. The pictures were pseudo-randomised such that pictures with similar content would appear in each of the above conditions equally. The cue was presented for $4000 \mathrm{~ms}$. The following picture was presented for 4000 ms. Lastly, a blank screen was presented for $6000-8200 \mathrm{~ms}$ (see Figure 1).

\subsubsection{Decision-making under uncertainty task:}

Participants were required to categorise whether a given circle would be larger or smaller than another hypothetical circle. Participants were instructed the following on the computer, 'In this experiment you will see different size circles. On the left or right you will see a circle. Opposite the circle you will see a \#. You will be asked to estimate whether the current circle will likely be bigger or smaller compared to another circle in the array. You will have to wait for 4 seconds before you give your answer using the keyboard.' Participants then viewed an array of all possible circle sizes for 20 seconds. The array consisted of seven grey circles that ranged 
from small to large (pixel dimensions: 33 × 33; $63 \times 63 ; 93 \times 93 ; 122 \times 123 ; 152 \times$ $151 ; 181 \times 181 ; 211 \times 211)$.

The task consisted of 42 trials ( 6 of each circle size). Experimental trials were randomised. The grey circle and \# (pixel dimension: $70 \times 81$ ) were counterbalanced to the left and right. The grey circle and \# were presented for $4000 \mathrm{~ms}$. The response slide was presented for $2000 \mathrm{~ms}$ and asked participants to 'Press S for smaller; Press B for bigger'. Lastly, a fixation screen was presented for 6000 - 7000 ms (see Figure 1).

INSERT FIGURE 1 ABOUT HERE

\subsection{Questionnaires}

STAI (Spielberger, Gorsuch, Lushene, Vagg, \& Jacobs, 1983) and IU questionnaires (Freeston, Rhéaume, Letarte, Dugas, \& Ladouceur, 1994) were measured.

\subsection{Behavioural data scoring}

Rating data were reduced for each participant by calculating their average responses for each experimental condition using the E-Data Aid tool in E-Prime (Psychology Software Tools Ltd, Pittsburgh, PA).

2.5.1 Associative threat learning task: Average expectancy ratings were calculated for the following conditions (Acquisition CS+, Acquisition CS-, Extinction Early CS+, Extinction Early CS-, Extinction Late CS+, Extinction Late CS-). 
2.5.2 Decision-making under uncertainty task: Reaction times under $200 \mathrm{~ms}$ were discarded. Remaining reaction times greater or equal to $200 \mathrm{~ms}$ were z--scored to control for interindividual differences in reaction time. The reaction times were averaged for each trial type (Circles 1-7, from small to large) and then collapsed over the following conditions: Certain (Circles 1 and 7), Mildly Uncertain (Circles 2 and 6), Quite Uncertain (Circles 3 and 5) and Very Uncertain (Circle 4).

Choices were coded into 1 and 0 , stimuli that were predicted as bigger were assigned a value of 1 and stimuli that were predicted as smaller were assigned a value of 0 . Average values for each condition (Circles 1-7, from small to large) essentially acted as subjects' probability of stimulus size. For example, a value of 1 , meant that the subject always picked bigger, whilst a value of 0.5 meant that the subject picked between bigger and smaller equally.

\subsection{Physiological acquisition}

Physiological recordings were obtained using AD Instruments (AD Instruments Ltd, Chalgrove, Oxfordshire) hardware and software.

Electrodermal activity was measured with dry MLT116F silver/silver chloride bipolar finger electrodes that were attached to the distal phalanges of the index and middle fingers of the non-dominant hand. A low constant-voltage AC excitation of 22 $m V_{\text {rms }}$ at $75 \mathrm{~Hz}$ was passed through the electrodes, which were connected to a ML116 GSR Amp, and converted to DC before being digitized and stored. An ML138 Bio Amp connected to an ML870 PowerLab Unit Model 8/30 amplified the skin conductance signal, which was digitized through a 16-bit A/D converter at $1000 \mathrm{~Hz}$.

Facial EMG measurements of the corrugator supercilii muscles were obtained by using two pairs of $4 \mathrm{~mm} \mathrm{Ag/AgCl}$ bipolar surface electrodes connected to the 
ML138 Bio Amp. The centres of each pair of bipolar surface electrodes were approximately $15 \mathrm{~mm}$ apart. The reference electrode was a singular $8 \mathrm{~mm} \mathrm{Ag} / \mathrm{AgCl}$ electrode, placed upon the middle of the forehead, and connected to the ML138 Bio Amp. Before placing the EMG sensors the skin site was cleaned with distilled water and slightly abraded with isopropyl alcohol skin prep pads, to reduce skin impedance to an acceptable level (below $20 \mathrm{k} \Omega$ ). EMG was sampled at $1000 \mathrm{~Hz}$.

\subsection{Physiological scoring}

The physiological parameters were extracted using AD Instruments software. The same criteria were used across tasks for extracting skin conductance responses (SCR) and corrugator supercilii activity.

SCR was scored when there was an increase of skin conductance level exceeding 0.03 microSiemens (Dawson et al., 2000). The amplitude of each response was scored as the difference between the onset and the offset (peak). SCR onsets and offsets were counted if the SCR onset was within 0.5-3.5 seconds following stimlus onset (Morriss, Chapman, Tomlinson, \& Van Reekum, 2018). Trials with no discernible SCRs were scored as zero. SCR magnitudes were square root transformed to reduce skew and were z-scored to control for interindividual differences in skin conductance responsiveness (Ben-Shakhar, 1985).

A high-pass filter at $20 \mathrm{hz}$ was applied to the raw corrugator online (Solnik, DeVita, Rider, Long, \& Hortobágyi, 2008). The corrugator signal was root mean squared offline (Fridlund \& Cacioppo, 1986). No moving average filter was applied. Baseline corrected second by second means over the course of the stimulus (4 seconds) was extracted for corrugator supercilii. Baseline mean values were taken from the 2 second period before each trial began. 
2.7.1 Associative threat learning task: CS+ unpaired and CS- trials were included in the analysis, but CS+ paired trials were discarded to avoid sound confounds. SCR magnitudes were calculated by averaging SCR transformed values and zeros from the CS for each condition (Acquisition CS+, Acquisition CS-, Extinction Early CS+, Extinction Early CS-, Extinction Late CS+, Extinction Late CS-).

The corrugator supercilii was extracted from the CS for each condition (Acquisition CS+, Acquisition CS-, Extinction Early CS+, Extinction Early CS-, Extinction Late CS+, Extinction Late CS-).

2.7.2 Threat uncertainty task: SCR onsets and offsets were counted following cue and picture onset for the following conditions (Cue Certain Negative, Cue Certain Neutral, Cue Uncertain, Certain Negative Picture, Certain Neutral Picture Uncertain Negative Picture, Uncertain Neutral Picture).

Baseline corrected second by second means over the course of the cue and picture (4 seconds) were extracted for corrugator supercilii. The corrugator supercilii was extracted for each condition (Cue Certain Negative, Cue Certain Neutral, Cue Uncertain, Certain Negative Picture, Certain Neutral Picture Uncertain Negative Picture, Uncertain Neutral Picture).

2.7.3 Decision-making under uncertainty task: SCR onsets and offsets were counted following the decision array. SCR magnitudes were calculated by averaging SCR transformed values and zeros for each condition (Circles 1-7, from small to large). 
Baseline corrected second by second means over the course of the decision array (4 seconds) were extracted for corrugator supercilii. The corrugator supercilii was extracted for each condition (Circles 1-7. from small to large).

For SCR and corrugator supercilii, the trial types were collapsed into: Certain (Circles 1 and 7), Mildly Uncertain (Circles 2 and 6), Quite Uncertain (Circles 3 and 5) and Very Uncertain (Circle 4).

\subsection{Behaviour and physiology analysis}

The analyses were conducted using the mixed procedure in SPSS 21.0 (SPSS, Inc; Chicago, Illinois). For all models a diagonal covariance matrix was used for level 1. Random effects included a random intercept for each individual subject, where a variance components covariance structure was used. A maximum likelihood estimator and the least significance difference procedure for pairwise comparisons was used for the multilevel models. Both IU and STAI were entered into the multilevel models as continuous variables.

Any interaction with IU or STAI was followed up with pairwise comparisons of the means between the conditions for IU or STAI estimated at the specific values of + or - 1 SD of mean (Morriss, Macdonald \& van Reekum, 2016; Morriss, McSorely \& van Reekum, 2017). The + or - $1 S D$ of mean data are estimated from the multilevel model of the entire sample, not unlike performing a simple slopes analysis in a multiple regression analysis.

2.8.1 Associative threat learning task: Separate multilevel models were conducted on expectancy ratings and SCR magnitude for each phase (Acquisition, Extinction). For expectancy ratings and SCR magnitude during the acquisition phase, Stimulus 
(CS+, CS-) was entered at level 1 and individual subjects at level 2. For expectancy ratings and SCR magnitude during the extinction phase, Stimulus (CS+, CS-) and Time (Early, Late) were entered at level 1 and individual subjects at level 2. The models for the corrugator supercilii in acquisition included Stimulus (CS+, CS-) and Second $(1,2,3,4)$ at level 1 and individual subjects at level 2 . In addition, the model for corrugator supercilii in extinction included Stimulus (CS+, CS-), Time (Early, Late) and Second $(1,2,3,4)$ at level 1 and individual subjects at level 2 .

2.8.2 Threat uncertainty task: SCR magnitude to the cue during the threat uncertainty task was assessed by including Cue (Certain Negative, Certain Neutral and Uncertain) at level 1 and individual subjects at level 2. To examine the impact of cue on SCR magnitude to the picture, an additional model was conducted, where Cue (Certain, Uncertain) and Picture (Negative, Neutral) was entered at level 1 and individual subjects at level 2. Furthermore, corrugator supercilii activity to the cue was assessed by including Cue (Certain Negative, Certain Neutral and Uncertain) and Second $(1,2,3,4)$ at level 1 and individual subjects at level 2. Lastly, to assess the impact of cue on corrugator supercilii activity during the picture, another model was conducted where Cue (Certain, Uncertain), Picture (Negative, Neutral) and Second $(1,2,3,4)$ were entered at level 1 and individual subjects at level 2 .

\subsubsection{Decision-making under uncertainty task: Choices to the decision array were} assessed by including Levels of Uncertainty (Circles 1-7, from small to large) at level 1 and individual subjects at level 2 .

Reaction time and SCR magnitude to the decision array was assessed by including Levels of Uncertainty (Certain, Mildly Uncertain, Quite Uncertain and Very 
Uncertain) at level 1 and individual subjects at level 2. Furthermore, corrugator supercilii activity to the decision array was assessed by including Levels of Uncertainty (Certain, Mildly Uncertain, Quite Uncertain and Very Uncertain) and Second $(1,2,3,4)$ at level 1 and individual subjects at level 2.

\section{Results}

\subsection{Questionnaires}

Similar distributions and internal reliability of scores were found for the self-report anxiety measures, STAI $(M=45.18 ; S D=9.09$; range $=26-66 ; \alpha=0.88), \mathrm{IU}(M=$ $61.82 ; S D=18.02 ;$ range $=33-103 ; \alpha=0.93$ )

\subsection{Associative threat learning task}

3.2.1 Ratings: Participants rated the sound stimulus as aversive $(M=2.14, S D=1.3$, range $1-6$, where $1=$ very negative and $9=$ very positive $)$ and arousing $(M=7.19$, $S D=1.4$, range $3-9$ where $1=$ calm and $9=$ excited $)$.

For the expectancy ratings, during acquisition participants reported greater expectancy of the sound with the CS+, compared to CS- [Stimulus: $F(1,79.747)=$ 119.416, $p<0.001$ ] (for descriptive statistics see Table 1). No other significant interactions with IU group or STAI were found for the ratings during acquisition, max $F=0.730$.

During extinction, participants reported greater expectancy of the sound with the CS+, compared to CS- [Stimulus: $F(1,121.742)=61.407, p<0.001$ ]. The expectancy ratings dropped over time [Time: $F(1,121.742)=5.274, p=0.023$; Stimulus $x$ Time: $F(1,121.742)=9.878, p=0.002$ ]. Follow-up pairwise comparisons 
revealed that the expectancy rating of the sound with the CS+ dropped significantly from early to late extinction, $p<0.001$. However, the expectancy rating of the CSwith the sound remained low and did not change with time, $p=0.546$. No other significant interactions with IU group or STAI were found for the ratings during extinction, $\max F=0.810$.

3.2.2 SCR: As expected, CS+ stimuli elicited larger SCR magnitudes than CSduring acquisition [Stimulus: $F(1,79.516)=16.134, p<.001$ ] (see, Table 1). There were no significant interactions between Stimulus x IU or STAI for SCR magnitude during acquisition, $\max F=1.092$.

During extinction, SCR magnitude was greater for the CS+ vs. CS- [Stimulus: $F(1,160.257)=14.186, p<0.001]($ see Table 1$)$. Additionally, SCR magnitude was greater for the CS+ vs. CS- during early extinction, $p<0.001$, but not for late extinction, $p=0.224$ [Stimulus $\times$ Time: $F(1,160.257)=4.253, p=0.041$ ].

As predicted IU was related to SCR magnitude during extinction [Stimulus $\mathrm{X}$ Time $\mathrm{x}$ IU interaction: $F(1,160.257)=6.760, p=0.010]$. Further inspection of followup pairwise comparisons showed that lower IU ( $1 S D$ below the mean) was associated with significantly greater SCR magnitude in early extinction to the CS+, relative to the CS-, $p<0.001$, but not in late extinction, $p=0.313$ (see, Figure. 2). In contrast, higher IU (1 SD above the mean) was associated with greater SCR magnitude to CS+ vs. CS- in both early extinction, $p=0.05$, and late extinction, $p=$ 0.011. Furthermore, low IU was associated with reduced SCR magnitude to the CS+ from early to late extinction, $p=0.012$, and an increase in SCR magnitude to the CSfrom early to late extinction, $p=0.047$. No other significant main effects or 
interactions were found with Stimulus, Time, IU or STAI for SCR magnitude during extinction, max $F=1.919$ (see Supplementary Material).

3.2.3 Corrugator supercilii activity: During acquisition larger corrugator supercilii activity was observed to the CS+ vs. CS- [Stimulus: $F(1,167.048)=19.865, p<$ 0.001] (see, Table 1). There were no significant interactions between Stimulus or Second $\mathrm{x}$ IU or STAI for corrugator supercilii activity during acquisition, $\max F=$ 2.202.

During extinction larger corrugator supercilii activity was observed to the CS+ vs. CS- at trend [Stimulus: $F(1,398.194)=3.356, p=0.068$ ] (see, Table 1).

Corrugator supercilii activity reduced across early to late extinction [Time:

$F(1,398.194)=9.256, p=0.003]$. As expected, individuals scoring higher in IU were shown to exhibit larger larger corrugator supercilii activity to the CS+vs. CS- during extinction, $p=0.02$, compared to individuals scoring lower in IU, $p=0.49$ [Stimulus $\mathrm{x}$ IU: $F(1,398.194)=6.438, p=0.012$ ] (see Figure 2 ). No other significant interactions between Stimulus or Second x IU or STAI were observed for corrugator supercilii activity during extinction, $\max F=2.911$.

INSERT TABLE 1 AND FIGURE 2 ABOUT HERE

\subsection{Threat uncertainty task}

3.3.2 SCR: Certain negative cues elicited larger SCR magnitudes, compared to the certain neutral cue and uncertain cue, $p$ 's $<0.001$ [Cue: $F(1,79.516)=16.134, p<$ 0.001] (see, Table 2). However, there was no significant difference between SCR magnitudes for certain neutral cues and uncertain cues, $p=0.334$. There were no 
significant interactions between Cue $\mathrm{x}$ IU or STAI for SCR magnitude, max $F=$ 0.685 .

As expected negative pictures elicited larger SCR magnitudes, compared to neutral pictures [Picture: $F(1,151.603)=13.369, p<0.001]$. No other significant interactions between Cue x Picture x IU or STAI for SCR magnitude were observed, $\max F=3.543$ (see Supplementary Material).

\subsubsection{Corrugator supercilii activity: No significant main effects or interactions} between Cue x IU or STAI for corrugator supercilii activity were observed, max $F=$ 1.242. Larger corrugator supercilii activity was found to negative, compared to neutral pictures [Picture: $F(1,397.444)=119.813, p<0.001$; Picture $\times$ Second: $F(1,397.444)=11.742, p<0.001]($ see Table 2$)$

An interaction between Cue, Picture and STAI emerged for corrugator supercilii activity [Cue $\times$ Picture $\times$ STAI interaction: $F(1,397.444)=14.956, p<$ 0.001] (see Figure 3). Follow-up pairwise comparisons revealed that higher STAI was associated with larger corrugator supercilii activity to both certain negative pictures, compared to certain neutral pictures, and uncertain negative pictures, compared to uncertain neutral pictures, $p$ 's $<0.001$. Moreover, higher STAI was associated with greater corrugator supercilii activity to certain neutral pictures, compared to uncertain neutral pictures, $p<0.001$, but there was no significant difference between certain negative pictures, compared to uncertain negative pictures, $p=0.383$. Lower STAI was associated with larger corrugator supercilii activity to certain negative pictures, compared to certain neutral pictures, $p<0.001$, but not to uncertain negative, compared to uncertain neutral pictures, $p=0.252$. In 
addition, lower STAI was associated with greater corrugator supercilii activity to certain pictures versus uncertain pictures, regardless of valence, $p$ 's $<0.015$.

No other significant interactions between Cue x Picture x IU or STAI for corrugator supercilii activity were observed, $\max F=3.514$.

INSERT TABLE 2 AND FIGURE 3 ABOUT HERE

\subsection{Decision-making under uncertainty task}

Three subjects missed responses to over half of the conditions and therefore were excluded from the analysis of the decision-making task.

3.4.1 Behavioural responses: Subjects predicted circle size as expected, such that there were significant differences in choices based on probability, particularly for circles in the middle (i.e. $2,3,4,5$ ), $p$ 's $<0.05$. Choices based on probability did not differ between circles 1 and 2, and circles 5, 6 and 7, p's > 0.05 [Levels of Uncertainty: $F(1,61.359)=33.646, p<0.001$ ] (see table 3$)$. For choices during the decision-making task there was a trend between Levels of Uncertainty and IU [Levels of Uncertainty $x$ IU interaction: $F(1,53.654)=2.147, p=0.054$ ]. Follow-up pairwise comparisons revealed that higher IU, relative to lower IU was associated with more accurate choice predictions of whether a circle would be likely bigger or smaller than the another circle shown in the array. For example, high IU was associated with better probabilistic choices for circles in the middle (i.e. $2,3,4)$ p's < 0.05 , whilst low IU was not, $p$ 's $>0.2$ (see Figure 4).

No other significant main effects or interactions between Levels of Uncertainty x IU or STAI for choices or reaction times were observed, max $F=.314$. 
3.4.2 SCR: For SCR magnitude an interaction at trend between Levels of Uncertainty and IU emerged [Levels of Uncertainty $x$ IU interaction: $F(1,42)=2.369$, $p=0.068]$ (see Figure 5). Follow-up pairwise comparisons revealed that lower IU was associated with greater SCR magnitude to very uncertain versus certain stimuli, $p=0.020$. No other significant main effects or interactions between Levels of Uncertainty $\times$ STAI for SCR magnitudes were observed, $\max F=0.798$.

3.4.3 Corrugator supercilii activity: IU was related to corrugator supercilii activity during the decision-making task [Levels of Uncertainty $\mathrm{x} I U$ interaction: $F(1,193.140)$ $=6.830, p<0.001$ ] (see Figure 4). Further inspection of follow-up pairwise comparisons revealed that higher IU was associated with significantly reduced corrugator supercilii activity to certain and mildy uncertain, relative to quite and very uncertain stimuli, $p$ 's $<0.032$. Lower IU was associated only with larger corrugator activity to the mildy uncertain relative to the very uncertain, $p=0.024$. No other significant interactions between Levels of Uncertainty $\mathrm{x}$ IU or STAI for the corrugator supercilii were observed, max $F=1.950$ (see Supplementary Material).

INSERT TABLE 3, FIGURE 4 AND FIGURE 5 ABOUT HERE

\subsection{Specificity of IU on anticipatory physiological responses across tasks}

Additional post hoc tests were conducted to assess the specificity of IU on anticipatory physiological responses across tasks. Difference scores from each task were calculated (associative learning task: Extinction (CS+ Early - CS-Early) - (CS+ Late - CS-Late); threat uncertainty task: (Negative Certain - Negative Uncertain) - (Neutral 
Certain - Neutral Uncertain); decision making under uncertainty task: (Certain+ Mildy Uncertain)/2 - (Quite Uncertain + Very Uncertain)/2). Separate regression analyses were performed for SCR and corrugator supercilii. IU (controlling for STAI) was entered as the dependent variable and the SCR and corrugator supercilii difference scores from the tasks were entered as independent variables.

3.5.1 SCR: The overall model was significant $\left[R^{2}=.236, F(3,38)=3.921\right.$, $p=0.016]$. Inspection of the beta-weights revealed that IU (controlling for STAI) was significantly related to the associative learning extinction phase, $(\beta=0.213, p=$ 0.013 ) but not the decision-making under uncertainty task $(\beta=-0.376, p=0.4)$ or threat uncertainty task ( $\beta=-0.122, p=0.4)$. A follow up hierarchal regression was performed to assess the specificity of the relationship of IU with the associative learning extinction phase. IU (controlling for STAI) was entered as the dependent variable and the decision-making under uncertainty and threat uncertainty tasks were entered at the first step and the associative learning extinction phase in the second step. The results revealed specificity of IU with the associative learning extinction phase over the other tasks, [First step: $R^{2}=.098, F(2,39)=2.126$, $p=0.133$, second step: $\left.\Delta R^{2}=.236, F(1,38)=6.872, p=0.013\right]$.

3.5.2 Corrugator supercilii activity: The overall model was not significant $\left[R^{2}=.078, F(3,38)=1.065, p=0.375\right]$.

\section{Discussion}

In the current study, IU was related to both SCR magnitude and corrugator supercilii activity for the associative threat learning and decision-making contexts. However, STAI was related to corrugator supercilii activity during the threat uncertainty context. Taken together this research helps us further dissect the role of IU on different 
aspects of anticipatory physiological responding (i.e. valence and arousal) during different contexts.

Typical patterns of threat acquisition were observed, such that larger SCR magnitude, corrugator supercilii activity and expectancy ratings were found for the learned threat vs. safety cues (for review see, Lonsdorf et al., 2017). In addition, effects of threat extinction were observed as larger SCR magnitude and expectancy ratings were found for the early part of the extinction phase, relative to the late part of extinction phase. Similar to previous work, higher IU was associated with reduced threat extinction, as shown by larger SCR magnitude and corrugator supercilii activity to learned threat vs. safety cues across the extinction phase (Morriss et al., 2015; Morriss, Christakou, et al., 2016; Morriss, Macdonald, et al., 2016; Morriss \& van Reekum, 2019). The observed IU-related effects on SCR magnitude and corrugator supercilii activity during extinction were specific to IU, over STAI. These findings provide further evidence that threat extinction may induce anxiety in individuals who score higher in IU because the contingencies are unknown.

In the threat uncertainty task, similar patterns of physiological responses to previous research were observed (Grupe et al., 2011). For example, larger SCR magnitude was found to the certain negative cue, relative to the certain neutral and uncertain cues. Additionally, larger SCR magnitude and corrugator supercilii activity was observed for negative, compared to neutral pictures (Lang \& Bradley, 2010; Lang, Greenwald, Bradley, \& Hamm, 1993). Higher STAI was associated with larger corrugator supercilii activity to negative images that followed uncertain and certain cues, whilst lower STAI was associated with larger corrugator supercilii activity only to negative images that followed certain cues. Importantly, the observed STAIrelated effects on corrugator supercilii activity during the threat uncertainty task were 
specific to STAI, over IU. Although these results were not expected, the results aren't surprising given the lack of reported IU effects on physiological measures during these type of tasks (Bennett et al., 2018; Grupe \& Nitschke, 2011). There may be multiple reasons for this result: (1) the task is not that uncertain given the known contingencies and therefore is not as motivationally relevant to individuals who score high in IU, and (2) the task presents many different types of negative images, tapping into broader negative affective states, which may be more motivationally relevant for STAI, given that the items are relevant for both anxiety and depression (Grös, Antony, Simms, \& McCabe, 2007).

For the decision-making under uncertainty task, all participants gave the typical choices when predicting whether a hypothetical circle would likely be bigger or smaller than the one that was presented. Notably, high IU, relative to low IU was associated with better choice accuracy. This result may be interpreted to reflect differences in motivational relevance for high and low IU individuals. High IU individuals may have been more engaged in the task in order to reduce uncertainty. In addition, high IU individuals relative to low IU individuals exhibited reduced corrugator supercilii activity during the anticipation of making a decision to certain and mildy uncertain stimuli, compared to quite and very uncertain stimuli. This can be interpreted as high IU individuals expressing greater relief to stimuli that are relatively more certain and expressing distress to stimuli that are relatively more uncertain. Lastly, lower IU was associated with larger SCR magnitudes to the uncertain stimuli, relative to certain stimuli. Whilst this effect for SCR magnitude was in the opposite direction for higher IU, albeit not significant. These results are difficult to interpret, as heightened arousal to uncertainty would be expected for high IU. However, given the corrugator supercilii results with IU during the decision-making 
task, these changes in SCR magnitude may reflect arousal from liking uncertain stimuli in low IU individuals. Additional research is needed to clarify the extent to which arousal in this decision-making task is related to liking/distress.

The results from the decision-making task are in line with previous work related to IU, showing that high IU individuals may seek to reduce uncertainty (Jacoby et al., 2014; Jacoby et al., 2016; Ladouceur et al., 1997), and may feel relief from certainty. Previous work has shown individual differences in IU to modulate decision-making during tasks with valenced outcomes (Carleton et al., 2016; Luhmann et al., 2011; Tanovic, Hajcak, et al., 2018; Tanovic, Pruessner, \& Joormann, 2018). Here it was shown that individual differences in IU modulate decision-making in the absence of valenced outcomes or consequences, thus suggesting that anticipating making a decision under uncertainty is enough to induce heightened physiological responses. However, it should be noted that the decisionmaking task used in this study relied on other processes such as working memory (i.e. remembering the stimulus array at the beginning of the task). Therefore, it is possible that the IU effects observed may be specifically related to making uncertain decisions based on memory.

IU was specifically related to the SCR magnitude during the associative threat extinction phase, over the decision-making and threat uncertainty tasks. However, no IU-related specificity was found for the corrugator supercilii activity between tasks. SCR is thought to capture differences in arousal (Dawson, Schell, \& Filion, 2000) and corrugator supercilli activity is thought to capture differences in valence, particularly negative affect (Tassinary, Cacioppo, \& Vanman, 2007). The specificity of IU and SCR magnitude results suggest that IU may be more relevant for modulating arousal related anticipation during contexts where the contingencies are 
unknown and require updating such as threat extinction. IU may not be as relevant for aspects of arousal related anticipation during situations where the rules of uncertainty are known and during the initial learning of threat associations, as in the threat uncertainty task and the associative learning acquisition phase. The lack of specificity for IU and corrugator supercilii activity suggests that valence related effects and IU may be more general i.e. high IU individuals may find uncertainty negative regardless of the context. Taken together these findings are in line with Carleton's (2016b) definition of IU, where the unknown serves as the ultimate stressor. Further work is needed to examine how physiological profiles during different contexts are related to different levels of uncertainty/unknowns in the absence and presence of valenced outcomes (Shihata et al., 2016). Furthermore, it will be important to examine how physiological profiles during different contexts are related to the different IU subscales (Carleton et al., 2007), as this may reveal distinct underlying mechanisms that serve as common denominators across IUrelated psychopathology (see Supplementary Material for additional analyses). Ultimately, this body of research will feed into developing future transdiagnostic models of IU.

The current study had a number of shortcomings. Firstly, the contexts that were examined were based on the most popular areas of research on uncertainty (Morriss, Gell \& van Reekum, 2019).There may have been other contexts that would be relevant to address and which have been missed here (i.e. attentional inhibition, perception). Furthermore, there may have been more elegant ways to subdivide the different contexts by levels of uncertainty. For example, uncertain outcomes of known risk versus uncertain outcomes where the probabilities are unknown. Secondly, the use of different stimuli to induce threat likely varied in aversiveness, 
thus findings between the associative learning and threat uncertainty task may be due to the level of aversiveness rather than the level of uncertainty. Thirdly, the study did not include other measures that are typically used to assess anticipation (i.e. heart rate variability and the CNV or SPN component). Fourthly, some of multilevel model analyses revealed opposite results for both IU and STAI, which may have been due to multicollinearity between the two questionnaires (see Supplementary Material for additional analyses). Lastly, the experiment was conducted on a relatively small sample from the UK as a proof of concept. To assess the robustness of these effects future research should aim to use larger, more diverse and rigorously screened samples from around the world.

In conclusion, these initial results provide insight into how IU is associated with different aspects of anticipation (i.e. valence and arousal) during different contexts, which will be relevant for understanding IU and potential treatment targets (Carleton, 2016a, 2016b; Grupe \& Nitschke, 2013). Further research is needed to explore how individual differences in IU modulate different aspects of anticipatory physiological responding during contexts with varying levels of uncertainty/unknowns and valence outcomes. 


\section{Author Note}

This research was supported by a: (1) NARSAD Young Investigator Grant from the Brain \& Behavior Research Foundation (27567), (2) an ESRC New Investigator Grant (ES/R01145/1) and (3) a British Academy Small Research Grant (SG163121) awarded to Jayne Morriss. The research was conducted at the Centre for Integrative Neuroscience and Neurodynamics (CINN) at the University of Reading. The author wishes to thank: (1) Carien van Reekum for the use of lab facilities and feedback, (2) Francesco Saldarini, Alberto Dalla Verde and Kelsey Britton for their help in data collection and marking, and (3) Michael Lindner for help with Matlab scripts.

Furthermore, the author would like to thank the participants who took part in this study. The author states no conflict of interest. To access the data, please contact Dr. Jayne Morriss. 


\section{References}

Ben-Shakhar, G. (1985). Standardization within individuals: A simple method to neutralize individual differences in skin conductance. Psychophysiology, 22(3), 292-299. https://doi.org/10.1111/j.1469-8986.1985.tb01603.x

Bennett, K. P., Dickmann, J. S., \& Larson, C. L. (2018). If or when? Uncertainty's role in anxious anticipation. Psychophysiology, e13066. https://doi.org/10.1111/psyp.13066

Bradley, M. M., \& Lang, P. J. (2000). Emotion and motivation. Handbook of psychophysiology, 2, 602-642.

Carleton, R. N. (2016a). Fear of the unknown: One fear to rule them all? Journal of Anxiety Disorders, 41, 5-21. https://doi.org/10.1016/j.janxdis.2016.03.011

Carleton, R. N. (2016b). Into the unknown: A review and synthesis of contemporary models involving uncertainty. Journal of Anxiety Disorders, 39, 30-43. https://doi.org/10.1016/j.janxdis.2016.02.007

Carleton, R. N., Duranceau, S., Shulman, E. P., Zerff, M., Gonzales, J., \& Mishra, S. (2016). Self-reported intolerance of uncertainty and behavioural decisions. Journal of Behavior Therapy and Experimental Psychiatry, 51, 58-65. https://doi.org/10.1016/j.jbtep.2015.12.004

Carleton, R. N., Norton, M. P. J., \& Asmundson, G. J. (2007). Fearing the unknown: A short version of the Intolerance of Uncertainty Scale. Journal of anxiety disorders, 21(1), 105-117. https://doi.org/10.1016/j.janxdis.2006.03.014

Chin, B., Nelson, B. D., Jackson, F., \& Hajcak, G. (2016). Intolerance of uncertainty and startle potentiation in relation to different threat reinforcement rates. International Journal of Psychophysiology, 99, 79-84. https://doi.org/10.1016/j.ijpsycho.2015.11.006 
Dawson, M. E., Schell, A. M., \& Filion, D. L. (2000). The Electrodermal System. In J. T. Cacioppo, L. G. Tassinary, \& G. G. Berntson (Eds.), Handbook of Physiology (2nd ed., pp. 200-223). Cambridge, UK: Cambridge University Press.

Dunsmoor, J. E., Campese, V. D., Ceceli, A. O., LeDoux, J. E., \& Phelps, E. A. (2015). Novelty-facilitated extinction: providing a novel outcome in place of an expected threat diminishes recovery of defensive responses. Biological Psychiatry, 78(3), 203-209. https://doi.org/10.1016/j.biopsych.2014.12.008

Fergus, T. A., Bardeen, J. R., \& Wu, K. D. (2013). Intolerance of uncertainty and uncertainty-related attentional biases: evidence of facilitated engagement or disengagement difficulty? Cognitive Therapy and Research, 37(4), 735-741.

Fergus, T. A., \& Carleton, R. N. (2016). Intolerance of uncertainty and attentional networks: Unique associations with alerting. Journal of Anxiety Disorders, 41, 59-64. https://doi.org/10.1007/s10608-012-9509-9

Freeston, M. H., Rhéaume, J., Letarte, H., Dugas, M. J., \& Ladouceur, R. (1994). Why do people worry? Personality and Individual Differences, 17(6), 791-802. https://doi.org/10.1016/0191-8869(94)90048-5

Fridlund, A. J., \& Cacioppo, J. T. (1986). Guidelines for human electromyographic research. Psychophysiology, 23(5), 567-589. https://doi.org/10.1111/j.14698986.1986.tb00676.x

Gentes, E. L., \& Ruscio, A. M. (2011). A meta-analysis of the relation of intolerance of uncertainty to symptoms of generalized anxiety disorder, major depressive disorder, and obsessive-compulsive disorder. Clinical Psychology Review, 31(6), 923-933. https://doi.org/10.1016/j.cpr.2011.05.001 
Gole, M., Schäfer, A., \& Schienle, A. (2012). Event-related potentials during exposure to aversion and its anticipation: the moderating effect of intolerance of uncertainty. Neuroscience Letters, 507(2), 112-117. https://doi.org/10.1016/j.neulet.2011.11.054

Grös, D. F., Antony, M. M., Simms, L. J., \& McCabe, R. E. (2007). Psychometric properties of the State-Trait Inventory for Cognitive and Somatic Anxiety (STICSA): comparison to the State-Trait Anxiety Inventory (STAI). Psychological assessment, 19(4), 369. https://psycnet.apa.org/doi/10.1037/1040-3590.19.4.369

Grupe, D. W., \& Nitschke, J. B. (2011). Uncertainty is associated with biased expectancies and heightened responses to aversion. Emotion, 11(2), 413. https://psycnet.apa.org/doi/10.1037/a0022583

Grupe, D. W., \& Nitschke, J. B. (2013). Uncertainty and anticipation in anxiety: an integrated neurobiological and psychological perspective. Nature Reviews Neuroscience, 14(7), 488-501. https://doi.org/10.1038/nrn3524

Hartley, C. A., \& Phelps, E. A. (2012). Anxiety and decision-making. Biological Psychiatry, 72(2), 113-118. https://doi.org/10.1016/j.biopsych.2011.12.027 Jacoby, R. J., Abramowitz, J. S., Buck, B. E., \& Fabricant, L. E. (2014). How is the Beads Task related to intolerance of uncertainty in anxiety disorders? Journal of Anxiety Disorders, 28(6), 495-503. https://doi.org/10.1016/j.janxdis.2014.05.005

Jacoby, R. J., Abramowitz, J. S., Reuman, L., \& Blakey, S. M. (2016). Enhancing the ecological validity of the Beads Task as a behavioral measure of intolerance of uncertainty. Journal of Anxiety Disorders, 41, 43-49. https://doi.org/10.1016/j.janxdis.2016.02.003 
Jacoby, R. J., Reuman, L., Blakey, S. M., Hartsock, J., \& Abramowitz, J. S. (2017). "What if I make a mistake?": Examining uncertainty-related distress when decisions may harm oneself vs. others. Journal of Obsessive-Compulsive and Related Disorders. https://doi.org/10.1016/j.jocrd.2017.11.003

Ladouceur, R., Talbot, F., \& Dugas, M. J. (1997). Behavioral expressions of intolerance of uncertainty in worry: Experimental findings. Behavior Modification, 21(3), 355-371. https://doi.org/10.1177\%2F01454455970213006

Lang, P. J., \& Bradley, M. M. (2010). Emotion and the motivational brain. Biological Psychology, 84(3), 437-450. https://doi.org/10.1016/j.biopsycho.2009.10.007

Lang, P. J., Bradley, M. M., \& Cuthbert, B. N. (2005). International affective picture system (IAPS): Affective ratings of pictures and instruction manual: $\mathrm{NIMH}$, Center for the Study of Emotion \& Attention.

Lang, P. J., Greenwald, M. K., Bradley, M. M., \& Hamm, A. O. (1993). Looking at pictures: affective, facial, visceral, and behavioral reactions. Psychophysiology, 30(3), 261-273.

Lonsdorf, T. B., Menz, M. M., Andreatta, M., Fullana, M. A., Golkar, A., Haaker, J., . . Kruse, O. (2017). Don't fear 'fear conditioning': Methodological considerations for the design and analysis of studies on human fear acquisition, extinction, and return of fear. Neuroscience \& Biobehavioral Reviews, 77, 247-285. https://doi.org/10.1016/j.neubiorev.2017.02.026

Lonsdorf, T. B., \& Merz, C. J. (2017). More than just noise: Inter-individual differences in fear acquisition, extinction and return of fear in humansBiological, experiential, temperamental factors, and methodological pitfalls. Neuroscience \& Biobehavioral Reviews, 80, 703-728. https://doi.org/10.1016/j.neubiorev.2017.07.007 
Lucas, K., Luck, C. C., \& Lipp, O. V. (2018). Novelty-facilitated extinction and the reinstatement of conditional human fear. Behaviour Research and Therapy, 109, 68-74. https://doi.org/10.1016/j.brat.2018.08.002

Luhmann, C. C., Ishida, K., \& Hajcak, G. (2011). Intolerance of uncertainty and decisions about delayed, probabilistic rewards. Behavior Therapy, 42(3), 378386. https://doi.org/10.1016/j.beth.2010.09.002

McEvoy, P. M., \& Mahoney, A. E. (2012). To be sure, to be sure: Intolerance of uncertainty mediates symptoms of various anxiety disorders and depression. Behavior Therapy, 43(3), 533-545. https://doi.org/10.1016/j.beth.2011.02.007

Morriss, J., Chapman, C., Tomlinson, S., \& Van Reekum, C. M. (2018). Escape the bear and fall to the lion: The impact of avoidance availability on threat acquisition and extinction. Biological Psychology, 138, 73-80. https://doi.org/10.1016/j.biopsycho.2018.08.017

Morriss, J., Christakou, A., \& Van Reekum, C. M. (2015). Intolerance of uncertainty predicts fear extinction in amygdala-ventromedial prefrontal cortical circuitry. Biology of Mood \& Anxiety Disorders, 5(1), 1. https://doi.org/10.1186/s13587015-0019-8

Morriss, J., Christakou, A., \& Van Reekum, C. M. (2016). Nothing is safe: Intolerance of uncertainty is associated with compromised fear extinction learning. Biological Psychology, 121, 187-193. https://doi.org/10.1016/j.biopsycho.2016.05.001

Morriss, J., Gell, M., \& van Reekum, C. M. (2018). The uncertain brain: A co-ordinate based meta-analysis of the neural signatures supporting uncertainty during different contexts. Neuroscience \& Biobehavioral Reviews, 96, 241-249. https://doi.org/10.1016/j.neubiorev.2018.12.013 
Morriss, J., Macdonald, B., \& van Reekum, C. M. (2016). What Is Going On Around Here? Intolerance of Uncertainty Predicts Threat Generalization. PloS one, 11(5), e0154494. https://doi.org/10.1371/journal.pone.0154494

Morriss, J., \& McSorley, E. (2019). Intolerance of uncertainty is associated with reduced attentional inhibition in the absence of direct threat. Behaviour research and therapy, 118, 1-6. https://doi.org/10.1016/j.brat.2019.03.011

Morriss, J., \& van Reekum, C. M. (2019). I feel safe when i know: Contingency instruction promotes threat extinction in high intolerance of uncertainty individuals. Behaviour Research and Therapy, 116, 111-118. https://doi.org/10.1016/j.brat.2019.03.004

Nelson, B. D., \& Shankman, S. A. (2011). Does intolerance of uncertainty predict anticipatory startle responses to uncertain threat? International Journal of Psychophysiology, 81(2), 107-115.

https://doi.org/10.1016/j.ijpsycho.2011.05.003

Schienle, A., Köchel, A., Ebner, F., Reishofer, G., \& Schäfer, A. (2010). Neural correlates of intolerance of uncertainty. Neuroscience letters, 479(3), 272-276. https://doi.org/10.1016/j.neulet.2010.05.078

Shihata, S., McEvoy, P. M., Mullan, B. A., \& Carleton, R. N. (2016). Intolerance of uncertainty in emotional disorders: What uncertainties remain? Journal of Anxiety Disorders, 41, 115-124. https://doi.org/10.1016/j.janxdis.2016.05.001

Solnik, S., DeVita, P., Rider, P., Long, B., \& Hortobágyi, T. (2008). Teager-Kaiser Operator improves the accuracy of EMG onset detection independent of signal-to-noise ratio. Acta of bioengineering and biomechanics/Wroclaw University of Technology, 10(2), 65. 
Somerville, L. H., Wagner, D. D., Wig, G. S., Moran, J. M., Whalen, P. J., \& Kelley, W. M. (2013). Interactions between transient and sustained neural signals support the generation and regulation of anxious emotion. Cerebral Cortex, 23(1), 49-60. https://doi.org/10.1093/cercor/bhr373

Spielberger, C. D., Gorsuch, R. L., Lushene, R., Vagg, P., \& Jacobs, G. (1983). Consulting Psychologists Press, Inc. 2». Palo Alto (CA).

Tanovic, E., Gee, D. G., \& Joormann, J. (2018). Intolerance of uncertainty: Neural and psychophysiological correlates of the perception of uncertainty as threatening. Clinical Psychology Review, 60, 87-99. https://doi.org/10.1016/j.cpr.2018.01.001

Tanovic, E., Hajcak, G., \& Joormann, J. (2018). Hating waiting: Individual differences in willingness to wait in uncertainty. Journal of Experimental Psychopathology, 9(1), 2043808718778982. https://doi.org/10.1177/2043808718778982

Tanovic, E., Pruessner, L., \& Joormann, J. (2018). Attention and anticipation in response to varying levels of uncertain threat: An ERP study. Cognitive, Affective, \& Behavioral Neuroscience, 1-14. https://doi.org/10.3758/s13415018-0632-2

Tassinary, L. G., Cacioppo, J. T., \& Vanman, E. J. (2007). The skeletomotor system: Surface electromyography. In J. T. Cacioppo, L. G. Tassinary, \& G. G. Berntson (Eds.), Handbook of psychophysiology (pp. 267-299). New York, NY, US: Cambridge University Press. 


\section{Figure Captions}

Fig 1. Image displaying the three different uncertainty tasks used in the experiment.

Fig 2. Bar graphs depicting IU estimated at + or - $1 S D$ of mean IU (controlling for STAI) from the multilevel model analysis for SCR magnitude and corrugator supercilii activity during extinction phase of the associative threat learning task. High IU, relative to low IU individuals were found to show heightened SCR magnitude and corrugator supercilii responding to the CS+ versus CS- cue during extinction. Bars represent standard error at + or $-1 S D$ of mean IU. Square root transformed and zscored SCR magnitude $(\mu S)$, skin conductance magnitude measured in microSiemens. Baseline corrected corrugator supercilii activity $(\mu \mathrm{V})$, measured in microVolts.

Fig 3. Bar graphs depicting STAl estimated at + or - 1 SD of mean STAI (controlling for IU) from the multilevel model analysis for corrugator supercilii activity during the threat uncertainty task. High STAI, relative to low STAI individuals were found to show larger corrugator supercilii activity to both certain and uncertain negative pictures, relative to certain and uncertain neutral pictures. Low STAI, relative to high STAI individuals were only found to show larger supercilii activity to certain negative versus certain neutral pictures, and not uncertain negative versus uncertain neutral pictures. Bars represent standard error at + or $-1 S D$ of mean STAI. Baseline corrected corrugator supercilii activity $(\mu \mathrm{V})$, measured in microVolts.

Fig. 4. Graph illustrating IU estimated at + or - $1 S D$ of mean IU (controlling for STAI) from the multilevel model analysis for choices made during the decision-making 
under uncertainty task. Higher IU relative to lower IU was associated with more accurate choice predictions of whether a circle would be likely bigger or smaller than another circle shown in the array. This effect was particularly evident at the most certain circles ( 1 and 7). Choices were coded into 1 and 0 , stimuli that were predicted as bigger were assigned a value of 1 and stimuli that were predicted as smaller were assigned a value of 0 . Average values for each condition (Circles 1-7, from small to large) acted as subjects' probability of stimulus size.

Fig 5. Bar graphs showing IU estimated at + or $-1 S D$ of mean IU (controlling for STAI) from the multilevel model analysis for SCR magnitude and corrugator supercilii activity during the decision-making under uncertainty task. High IU, relative to low IU individuals were found to show reduced corrugator supercilii activity to the certain and mildy uncertain conditions, compared to the quite and very uncertain conditions. Low IU individuals displayed larger SCR magnitude to the very uncertain condition, compared to the certain condition, whilst high IU individuals did not show any significant differences in SCR magnitude across conditions. Bars represent standard error at + or $-1 S D$ of mean IU. Square root transformed and z-scored SCR magnitude $(\mu \mathrm{S})$, skin conductance magnitude measured in microSiemens. Baseline corrected corrugator supercilii activity $(\mu \mathrm{V})$, measured in microVolts. 
Table 1. Associative threat learning task summary of means $(S D)$ for each dependent measure as a function of stimulus (CS+ and CS-), separately for acquisition, early extinction and late extinction.

\begin{tabular}{|c|c|c|c|c|c|c|}
\hline \multirow[t]{2}{*}{ Measure } & \multicolumn{2}{|c|}{ Acquisition } & \multicolumn{2}{|c|}{$\begin{array}{c}\text { Early } \\
\text { Extinction }\end{array}$} & \multicolumn{2}{|c|}{ Late Extinction } \\
\hline & $\mathrm{CS}_{+}$ & CS- & $\mathrm{CS}_{+}$ & CS- & $\mathrm{CS}_{+}$ & CS- \\
\hline $\begin{array}{l}\text { Square root transformed and z-scored SCR magnitude } \\
(\sqrt{\mu S})\end{array}$ & $\begin{array}{c}0.38 \\
(0.42)\end{array}$ & $\begin{array}{c}0.05 \\
(0.34)\end{array}$ & $\begin{array}{c}0.07 \\
(0.37)\end{array}$ & $\begin{array}{l}-0.23 \\
(0.28)\end{array}$ & $\begin{array}{l}-0.05 \\
(0.39)\end{array}$ & $\begin{array}{l}-0.15 \\
(0.37)\end{array}$ \\
\hline Corrugator supercilii activity $(\mu \mathrm{V})$ & $\begin{array}{c}0.76 \\
(1.63)\end{array}$ & $\begin{array}{c}0.29 \\
(0.70)\end{array}$ & $\begin{array}{c}0.49 \\
(1.42)\end{array}$ & $\begin{array}{c}0.25 \\
(0.87)\end{array}$ & $\begin{array}{c}0.17 \\
(0.75)\end{array}$ & $\begin{array}{c}0.14 \\
(0.56)\end{array}$ \\
\hline Expectancy rating (1-9) & $\begin{array}{c}6.96 \\
(1.68)\end{array}$ & $\begin{array}{l}2.50 \\
(2.12)\end{array}$ & $\begin{array}{c}4.64 \\
(2.11)\end{array}$ & $\begin{array}{l}2.23 \\
(2.26)\end{array}$ & $\begin{array}{c}3.45 \\
(2.06)\end{array}$ & $\begin{array}{c}2.42 \\
(2.46)\end{array}$ \\
\hline
\end{tabular}

Note: SCR magnitude $(\sqrt{ } \mu S)$, square root transformed and z-scored skin conductance magnitude measured in microSiemens. Baseline corrected corrugator supercilii activity $(\mu \mathrm{V})$, measured in microVolts. Expectancy ratings from 1-9, where 1 is don't expect and 9 is very much expect. 
Table 2. Threat uncertainty task summary of means $(S D)$ for each dependent measure as a function of the cue and picture period separately.

\begin{tabular}{|c|c|c|c|c|c|}
\hline Measure & \multicolumn{2}{|c|}{$\begin{array}{c}\text { Certain Negative } \\
\text { Cue } \\
\end{array}$} & \multicolumn{2}{|c|}{$\begin{array}{c}\text { Certain Neutral } \\
\text { Cue } \\
\end{array}$} & Uncertain Cue \\
\hline $\begin{array}{l}\text { Square root transformed and z- } \\
\text { scored SCR magnitude }(\sqrt{\mu} S)\end{array}$ & \multicolumn{2}{|c|}{$0.14(0.26)$} & \multicolumn{2}{|c|}{$-0.10(0.22)$} & $-0.15(0.28)$ \\
\hline \multirow[t]{3}{*}{ Corrugator supercilii activity $(\mu \mathrm{V})$} & \multicolumn{2}{|c|}{$-0.06(0.81)$} & \multicolumn{2}{|c|}{$-0.03(0.45)$} & $0.01(0.46)$ \\
\hline & \multicolumn{2}{|c|}{ Certain Cue } & \multicolumn{2}{|c|}{ Uncertain Cue } & \\
\hline & Negative & Neutral & Negative & Neutral & \\
\hline $\begin{array}{l}\text { Square root transformed and z- } \\
\text { scored SCR magnitude }(\sqrt{\mu S})\end{array}$ & $\begin{array}{c}0.06 \\
(0.35)\end{array}$ & $\begin{array}{l}-0.14 \\
(0.23)\end{array}$ & $\begin{array}{c}0.14 \\
(0.45)\end{array}$ & $\begin{array}{l}-0.05 \\
(0.40)\end{array}$ & \\
\hline Corrugator supercilii activity $(\mu \mathrm{V})$ & $\begin{array}{c}0.89 \\
(1.22)\end{array}$ & $\begin{array}{l}-0.04 \\
(0.63)\end{array}$ & $\begin{array}{c}0.74 \\
(1.20)\end{array}$ & $\begin{array}{c}0.05 \\
(0.74)\end{array}$ & \\
\hline
\end{tabular}

Note: SCR magnitude $(\sqrt{ } \mu S)$, square root transformed and z-scored skin conductance magnitude measured in microSiemens. Baseline corrected corrugator supercilii activity $(\mu \mathrm{V})$, measured in microVolts. 
Table 3. Decision-making under uncertainty task summary of means (SD) for each dependent measure as a function of stimulus (Circles 1-7, from small to large) and levels of uncertainty (Certain, Mildy Uncertain, Quite Uncertain, Very Uncertain) separately.

\begin{tabular}{|c|c|c|c|c|c|c|c|}
\hline Measure & 1 & 2 & 3 & 4 & 5 & 6 & 7 \\
\hline Choice & $\begin{array}{l}0.83 \\
(0.31)\end{array}$ & $\begin{array}{c}0.68 \\
(0.32)\end{array}$ & $\begin{array}{c}0.48 \\
(0.33)\end{array}$ & $\begin{array}{l}0.30 \\
(0.31)\end{array}$ & $\begin{array}{c}0.18 \\
(0.25)\end{array}$ & $\begin{array}{c}0.14 \\
(0.26)\end{array}$ & $\begin{array}{c}0.12 \\
(0.27)\end{array}$ \\
\hline Measure & Certain & $\begin{array}{r}\mathrm{Mi} \\
\text { Unc } \\
\end{array}$ & dy & $\begin{array}{r}\text { Qu } \\
\text { Unce }\end{array}$ & rtain & \multicolumn{2}{|c|}{ Very Uncertain } \\
\hline $\begin{array}{l}\text { Square root transformed and z- } \\
\text { scored SCR magnitude }(\sqrt{\mu S})\end{array}$ & $-0.007(0.22)$ & \multicolumn{2}{|c|}{$-0.009(0.13)$} & \multicolumn{2}{|c|}{$-0.03(0.23)$} & \multicolumn{2}{|c|}{$0.03(0.37)$} \\
\hline Corrugator supercilii activity $(\mu \mathrm{V})$ & $-0.16(0.64)$ & \multicolumn{2}{|c|}{$-0.18(0.74)$} & \multicolumn{2}{|c|}{$-0.001(0.72)$} & \multirow{2}{*}{\multicolumn{2}{|c|}{$\begin{array}{c}-0.01(0.77) \\
0.06 \text { (0Ffigure } 1 \\
.42)\end{array}$}} \\
\hline Z-scored reaction time (ms) & $0.009(0.27)$ & \multicolumn{2}{|c|}{$-0.01(0.16)$} & \multicolumn{2}{|c|}{$-0.02(0.25)$} & & \\
\hline
\end{tabular}

Note: Choices were coded into 1 and 0 , stimuli that were predicted as bigger were assigned a value of 1 and stimuli that were predicted as smaller were assigned a value of 0 . Average values for each condition (Circles 1-7, from small to large) acted as subjects' probability of stimulus size. For example, a value of 1 , meant that the subject always picked bigger. SCR magnitude $(\sqrt{ } \mu S)$, square root transformed and zscored skin conductance magnitude measured in microSiemens. Baseline corrected corrugator supercilii activity $(\mu \mathrm{V})$, measured in microVolts. Reaction times $(\mathrm{ms}), \mathrm{z}$-scored reaction times measured in milliseconds. 